\title{
Summation of Powers in Open Resonator with Slotted Coupling Elements
}

\author{
Igor K. Kuzmichev, Bogdan I. Muzychishin, and Aleksey Y. Popkov \\ Department of Vacuum Electronics, O.Ya. Usikov Institute for Radiophysics and Electronics of the National Academy of Sciences of Ukraine, \\ Academician Proskura str., 12, 61085, Kharkiv, Ukraine
}

Corresponding author: Igor K. Kuzmichev (e-mail: kuzmichev.igr@i.ua).

\begin{abstract}
The paper analyzes the summation of the powers of two sources in a hemispherical open resonator (OR) during its tuning. The first higher axially asymmetric $\mathrm{TEM}_{10 q}$ oscillation mode is excited in the resonator. A circuit with an $E$ - tee waveguide is proposed, which makes it possible to research the summation of the powers using a Gunn diode. Studies of the conducting part of the millimeter range have been undertaken. It is shown that the coefficient of powers summation of two sources in the OR using slot coupling elements does not exceed $72 \%$. The use of one $H$ - polarized diffraction grating, which is in the resonator, does not lead to a significant increase in the summation coefficient when moving it. This is due to the excitation of the first type of $\mathrm{TEM}_{10 q}$ oscillations in the resonator.
\end{abstract}

INDEX TERMS Coupling element, diffraction grating, loaded $Q$-factor, oscillation mode, open resonator, powers summation, transmission coefficient.

\section{INTRODUCTION}

$\mathbf{R}$ ecently, great interest has arisen in the terahertz frequency range [1-5]. Since the waves of this range penetrate through paper, plastic, clothes, they can be used for inspection in crowded places using active or passive location methods. Terahertz waves are of interest for analyzing the spectra of individual stars and galaxies. Electromagnetic radiation of this range can penetrate biological tissue at short distances and be reflected, which makes it possible to diagnose some forms of cancer using safer, less aggressive, and painful procedures. To solve not only the listed, but also many other problems successfully, compact coherent terahertz radiation sources are required to provide output power levels sufficient for each specific practical application. However, as the frequency increases, the output power of both solid-state and classical vacuum sources decreases. In the first case, this is due to the increasing role of parasitic parameters of the device and shortening the time of charge carriers flight in the active region, and in the second one, with an increase in ohmic losses and a deterioration in the efficiency of interaction of the electron beam with one of the surface harmonics of the slowing down system with a decrease in the geometric dimensions of the device.

One of the possible ways to solve these problems is the use of power combiners. Bridge circuits and the summation of the powers of the individual sources in a single resonant volume in the microwave range are most widely used [6]. With the transition to the terahertz $(0.1<f(\mathrm{THz})<10)$ frequency range [7], the requirements for the accuracy of manufacturing the element base increase. On the other hand, to obtain a single-mode regime in cavity resonators, their geometric dimensions must be comparable to the operating wavelength. This leads to a decrease in the volume occupied by the operating oscillation, and, consequently, to the q-factor, which ultimately affects the powers summation coefficient of the individual sources. Therefore, in the indicated frequency ranges it is necessary to switch to open resonators (OR), which are used when adding the powers of solid-state sources in the EHF (extremely high-frequency) range [8-12]. The sources of oscillations are located in the resonant volume in almost all of these works. In this case, there is a strong coupling of the generators with the main resonator and, as a consequence, with each other. This causes difficulties in tuning the power combiner and makes such systems critical to the scatter of individual sources parameters, even with a small number of them [13]. In this regard, the most promising ones are ORs with sources removed from the resonant volume $[14,15]$. This ensures synchronization of active elements, and the connection between them becomes weaker. All summable sources are connected with resonator oscillation using slot coupling elements. In this case, the loaded $Q$-factor of the working oscillation will be significantly higher. This is of great practical importance when constructing power compressors [16] based on OR. The powers of several sources are added in the resonant volume. This power is output from the resonator in a time significantly less than the time for setting the oscillation in the OR. Due to this, it 
is possible to create pulse sources that do not have analogs in the indicated frequency range.

To sum up the powers of solid-state sources in OR, the basic mode of TEMooq oscillations is used $[10,11,15]$. In this case, all slotted coupling elements must be made within the spot of the field of the main oscillation of the resonator on a flat mirror. In our opinion, the most promising is the use of higher types of OR oscillations in the Hermite-Gauss functions for summing the powers of solid-state sources, as was done in [14]. Here, the first higher axially asymmetric mode of $\mathrm{TEM}_{10 q}$ oscillation characterized by two spots of the operating oscillation field on a flat mirror is excited in the resonator. A slotted coupling element is made in the center of each spot of the field on the mirror. The powers of the two sources are summed up in the resonator. The use of oscillation types $\mathrm{TEM}_{20 q}$ or $\mathrm{TEM}_{30 q}$ is not advisable. The use of these types of oscillations will allow to add up the powers of three $\left(\mathrm{TEM}_{20 q}\right.$ oscillation) or four (TEM $30 q$ oscillation) sources in the OR. However, in these cases, it is necessary to significantly increase the apertures of the resonator mirrors to obtain low diffraction losses, which will lead to an increase in the geometric dimensions of the power combiner itself.

Therefore, it is of practical interest to analyze the summation of the powers of two Gunn diodes in a hemispherical OR during its tuning. To date, no studies have been conducted on the effect of the distance between OR mirrors on the power summation factor of individual sources. It is difficult to find two Gann diodes that have the same operating frequencies and equal output power levels. Therefore, the study will use the original circuit on the $E$ tee waveguide. The first higher axially asymmetric mode of $\mathrm{TEM}_{10 q}$ oscillation is excited in the resonator. The relevance of these studies is also connected with the possibility of the summation of the powers in the OR at the harmonics of the fundamental frequency of the Gunn diodes. This will allow to creation compact terahertz sources, not only continuous, but also pulse action. In addition, it is advisable to consider the effect of a strip Hpolarized diffraction grating, located in the OR volume, on the behavior of the powers summation coefficient of two solid-state sources.

\section{EXPERIMENTAL STAND}

The block diagram of the experimental stand, using which studies were carried out on the summation of the powers of solid-state sources in a hemispherical OR, is shown in Fig. 1, and its appearance is shown in Fig. 2 and Fig. 3.

The OR is formed by flat mirror 19 with an aperture of 38 $\mathrm{mm}$ and spherical focusing mirror 22 with a curvature radius $R=39 \mathrm{~mm}$ and an aperture of $38 \mathrm{~mm}$. The flat mirror has two slotted coupling elements. The distance from the axis of this mirror to the center of each of the two slotted coupling elements is $3.5 \mathrm{~mm}$. Both coupling elements are smooth transitions 18 from a lowered section of $3.6 \mathrm{~mm} \times 0.15 \mathrm{~mm}$ to the main section of the waveguide $3.6 \mathrm{~mm} \times 1.8 \mathrm{~mm}$. With the help of these coupling elements, the first higher axially asymmetric mode of $\mathrm{TEM}_{10 q}$ oscillation is excited in the resonator. The signal is output from the resonator using one of two slotted coupling elements made on a spherical mirror. They also represent smooth transitions 24 from a lowered section of $3.6 \mathrm{~mm} \times 0.15 \mathrm{~mm}$ to the main section of the waveguide $3.6 \mathrm{~mm} \times 1.8 \mathrm{~mm}$. Short-circuit piston 25 is connected to the standard output of second waveguide 24 through a section of a curved waveguide 10 (see Fig. 3). The distance from the center of the spherical mirror to the centers of each of the two slotted coupling elements located on this mirror is $5.5 \mathrm{~mm}$. As in the case of a flat mirror $(3.5 \mathrm{~mm})$, this distance, is determined by the maximum value of the electric field strength of the $\mathrm{TEM}_{10 q}$ oscillation $(L / R \approx 0.6)$ at both resonator mirrors.

A 3A728B Gunn diode (see Fig. 1), operating at the second harmonic of the fundamental frequency is used as a solid-state source 1. The generator frequency is $74.935 \mathrm{GHz}$, the output power is $37 \mathrm{~mW}$. To expand the dynamic range $\mathrm{p}$ i-n modulator 2 is included in the circuit, with the help of which the EHF oscillations generated by the Gunn diode are modulated with a frequency of $1 \mathrm{kHz}$ from sound generator 3. To decouple the generator and the resonator, gate 4 is included in the circuit, the direct losses of which are $-0.53 \mathrm{~dB}$ at a frequency of $74.935 \mathrm{GHz}$, and the return losses are -7.6 $\mathrm{dB}$. The circuit also includes a directional coupler 5 to control the frequency of the Gunn oscillator. This path includes: section of waveguide 10, wave meter 11, detector 7, selective amplifier 8, and oscilloscope 9 (see Fig. 1). Directional coupler 5 also includes a path that makes it possible to measure the reflection coefficient $\Gamma$ from the resonator, which includes: polarization attenuator 6 , detector 7, selective amplifier 8 and oscilloscope 9 (see Fig. 1).

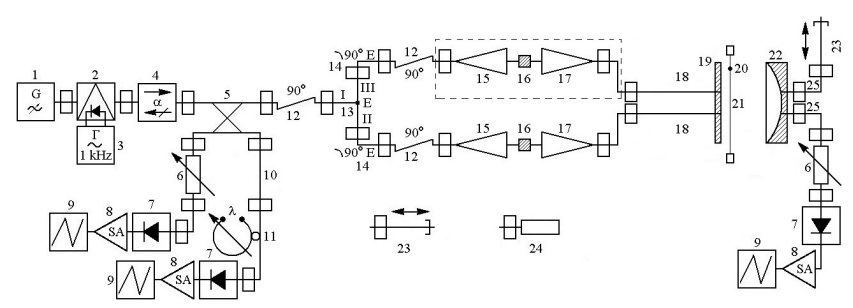

FIGURE 1. Block diagram of the experimental stand.

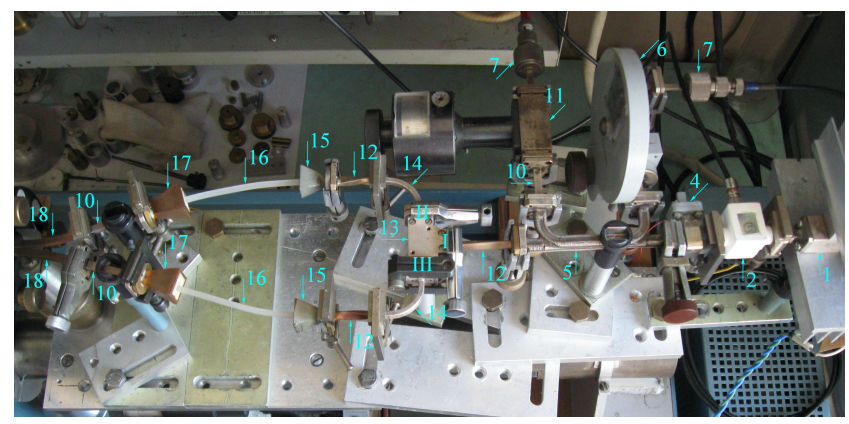

FIGURE 2. External view of the experimental stand without a resonator. 


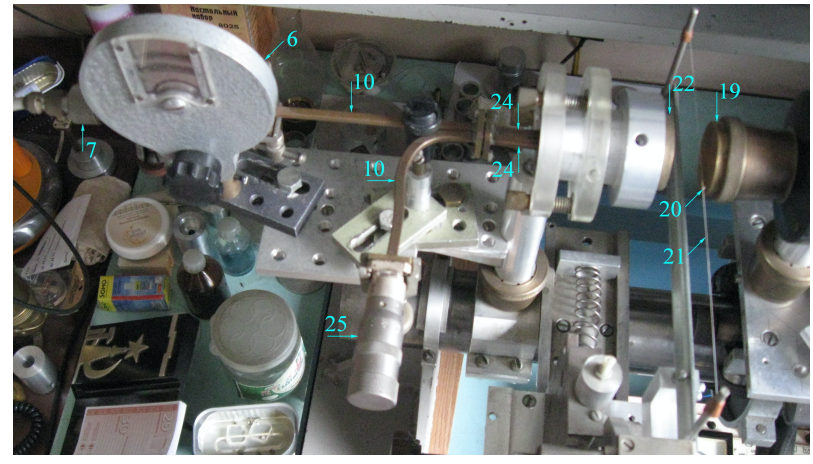

FIGURE 3. External view of the investigated hemispherical resonator.

A distinctive feature of the block diagram under consideration is that it allows to studying of the summation of the powers of two sources in the OR using one generator 1 . For this, waveguide $E$ - tee 13 is included in the circuit, which is connected to directional coupler 5 by waveguide twist 12 (see Fig. 1 and Fig. 2). For the TEM $10 q$ oscillation of the considered hemispherical OR, the electric field strengths in adjacent spots possess antiphase vectors [17].

If a signal is applied to the arm I of $E$ - tee waveguide 13 (wave $\mathrm{TE}_{10}$ of a rectangular waveguide), then the power of this wave will be divided equally between arms II and III. In this case, the electric fields of the TE10 waves in arms II and III will be antiphase at the same distance from the branching (for the considered $E$ - tee waveguide with a cross-section of $3.6 \mathrm{~mm} \times 1.8 \mathrm{~mm}$, this is $20 \mathrm{~mm}$ ). If now the waveguide curves in $E$ - plane 14, waveguide twists 12, pyramidal horns 15 , sections of dielectric waveguides 16 , pyramidal horns 17 , and sections of rectangular waveguides 10 will have the same length, connected to the arms II and III of the $E$ - tee waveguide, then the electric fields of the $\mathrm{TE}_{10}$ waves propagating along both paths (arms II and III) will be antiphase at the entrance to the resonator and should have the same amplitudes (see Fig. 1 and Fig. 2). Waveguide transitions 18 from a reduced cross-section to a standard one initially had the same lengths. And this is exactly what is needed to excite the $\mathrm{TEM}_{10 q}$ oscillations in the resonator with high efficiency. Dielectric waveguides 16 have a crosssection of $3.6 \mathrm{~mm} \times 2 \mathrm{~mm}$. The apertures of horns 15 are $14.5 \mathrm{~mm} \times 11.5 \mathrm{~mm}$ with a length of $19.5 \mathrm{~mm}$. Horn apertures 17 are $14.5 \mathrm{~mm} \times 10.5 \mathrm{~mm}$ with a length of 26.5 $\mathrm{mm}$.

The signal from the resonator is output using one of the two slot coupling elements 24 and through polarization attenuator 6 it is fed to detector 7, and then through selective amplifier 8 to oscilloscope 9 (see Fig. 1 and Fig. 3). To find the resonant transmission coefficient $K_{\text {transm, }}$ use the following procedure. After tuning the system to resonance with selective amplifier 8 , we fix the signal level at the output of the OR using measuring polarization attenuator 6 . Let it be equal to $N_{1}(\mathrm{~dB})$. After carrying out the entire cycle of measurements at the output of directional coupler 5 , we connect the receiving path consisting of polarization attenuator 6 , detector 7 , selective amplifier 8 , and a phase shifter instead of the $E$ - tee waveguide. Using the phase shifter, we achieve the maximum signal level on the dial gauge of selective amplifier 8. Changing the attenuation introduced into the path, using attenuator 6 , we achieve the same signal level on amplifier 8 . Let the signal level in this case be equal to $N_{2}(\mathrm{~dB})$. Then the field gain $K_{\text {transm }}=10^{-\Delta N / 20}$, where $\Delta N=N_{2}-N_{1}(\mathrm{~dB})$.

At the first stage, it is of practical interest to estimate the losses in the paths connected to arms II and III of the $E$ - tee waveguide. To do so matched load 23 is placed in the arm II of the waveguide $E$ - tee after the waveguide bending in $E$ plane 14 and waveguide twisting 12 (see Fig. 1). The measurements were carried out using a panoramic VSWR and attenuation meter R1-69. The path includes elements starting with waveguide valve 4 and up to a smooth transition to a waveguide of standard cross-section 18 . The total losses of the specified path (arm III of the waveguide tee) are -5.5 $\mathrm{dB}$ at the frequency of the Gunn generator of $74.935 \mathrm{GHz}$. As the next step, matched load 23 is connected to arm III of the $E$ - tee waveguide after waveguide bend 14 and waveguide twisting 12 . The total losses in the waveguide path, in this case, starting with waveguide valve 4 and up to the transition to the waveguide of standard section 18 , are $-5.6 \mathrm{~dB}$. Thus, an important practical conclusion can be drawn based on the analysis performed. With the same geometric length of both paths, the losses in them are almost the same. Therefore, using a measuring installation, the block diagram of which is shown in Fig. 1, and the appearance is in Fig. 2 and Fig. 3, it is possible to carry out studies on the summation of the powers of two sources in the OR using one master oscillator. In this case, the electric fields of the $\mathrm{TE}_{10}$ waves propagating along both paths (arms II and III of the $E$ tee waveguide) at the entrance to the resonator will have almost the same amplitudes and will be antiphase.

\section{STUDY OF THE SUMMATION OF POWERS IN OR}

There is a matched load 23 in arm II of the $E$ - tee waveguide after the waveguide bending in $E$ - plane 14 and waveguide twisting 12. Short-circuiting piston 25 is connected to the output of rectangular waveguide segment 10 which is a part of the waveguide path (see Fig. 1). The measurement results are shown in Fig. 4 (curve 2). As can be seen from the graph above, the resonant transmission coefficient $K_{\text {transm }}^{\text {(III) }}$ increases with decreasing distance between the OR mirrors.

This is due to a decrease in the diffraction losses for the considered oscillation as the cavity mirrors approach each other. The sharp drop in $K_{\text {transm }}^{\text {(II) }}$ at $L / R=0.49$ is associated with the semi-confocal geometry of the resonator under consideration. In this case, the $\mathrm{TEM}_{109}$ oscillation interacts with the higher $\mathrm{TEM}_{309}$ oscillation of the resonator, which has the same class of symmetry as our oscillation. At $L / R=$ 0.331, the TEM $\mathrm{T}_{106}$ oscillation interacts with another higher 
oscillation, which is excited in a hemispherical resonator using one slotted coupling element. That also leads to a decrease in $K_{\text {transm }}^{\text {(III) }}$.

Now we change the scheme of the experimental setup. There is matched load 23 (see Fig. 1) in arm III of the $E$ - tee waveguide after waveguide bending in E-plane 14 and waveguide twisting 12. Short-circuiting piston 25 is connected to the output of a section of rectangular waveguide 10 which is a part of the waveguide path. The resonator is excited through the second slotted coupling element linked to the waveguide path connected to arm II of the $E$ - tee waveguide. The measurement results are shown in Fig. 4 (curve 3).

The figure shows that the behavior $K_{\text {transm }}^{(\mathrm{II})}$ completely repeats the behavior $K_{\text {transm }}^{(\text {III) }}$ with a decrease in the distance between the cavity mirrors. The difference is that $K_{\text {transm }}^{(\mathrm{II})}$ is slightly less than $K_{\text {transm }}^{\text {(III) }}$ in the range of variation of the distances between the cavity mirrors $L / R=0.172 \div 0.651$. Apparently, this is due to the difference in the total losses in the paths including arms II $(-5.6 \mathrm{~dB})$ or III $(-5.5 \mathrm{~dB})$ of $E$ - tee waveguide 13 .

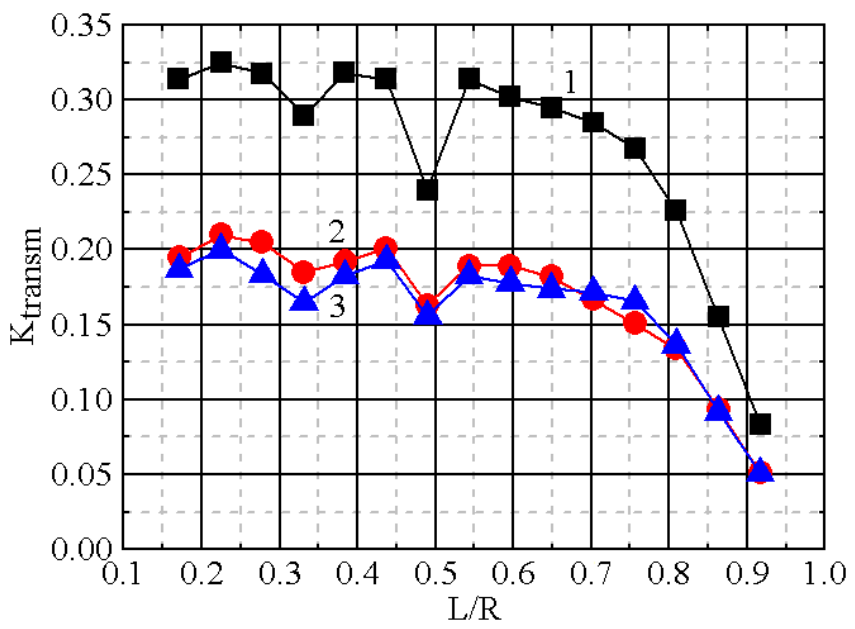

FIGURE 4. Dependences of the resonance transfer coefficients on the distance between the OR mirrors when the TEM $10 q$ oscillation is excited in different ways.

Now we excite the resonator with two slot coupling elements made on a flat mirror (see Fig. 1). The dependence of the resonant transfer coefficient $K_{\text {transm }}^{(\mathrm{I})}$ on the change in the normalized distance $L / R$ between the OR mirrors is shown in Fig. 4 (curve 1). The general behavior of $K_{\text {transm }}^{(\mathrm{I})}$ is similar to $K_{\text {transm }}^{(\mathrm{II})}$ and $K_{\text {transm }}^{(\mathrm{III})}$. The difference is that the resonant transfer coefficient becomes significantly higher over the entire tuning range of the resonator when the resonator is excited with two slot coupling elements.

Let us analyze how the power summation coefficient of the two sources behaves when the distance between the cavity mirrors changes. To find the power summation coefficient we use the formula $K_{\text {summ }}^{2}=$ $=\left[K_{\text {transm }}^{(\mathrm{I})}\right]^{2} /\left[\left(K_{\text {transm }}^{(\mathrm{II})}\right)^{2}+\left(K_{\text {transm }}^{(\mathrm{III})}\right)^{2}\right]$. The calculation results are shown in Fig. 5.

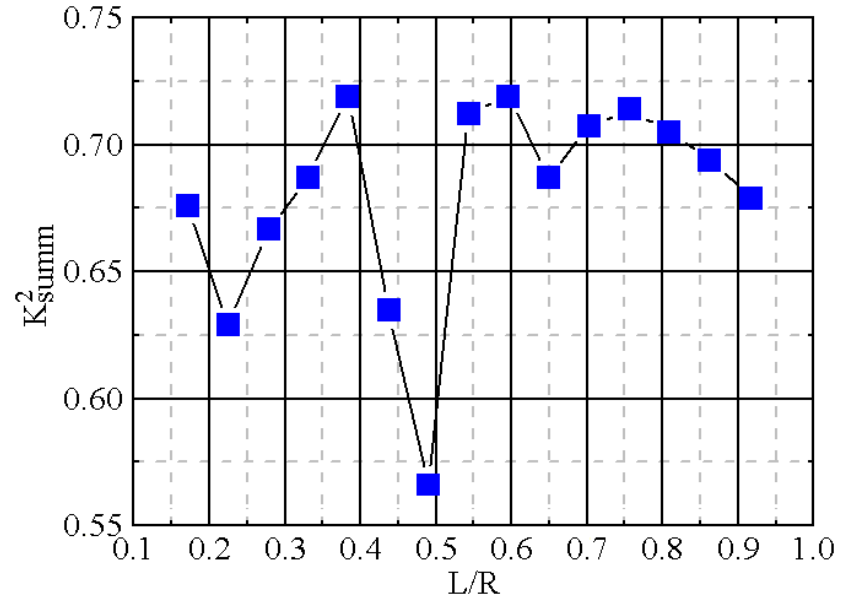

FIGURE 5. Behavior of the power summation factor in OR when the distance between the mirrors changes.

The figure shows that the maximum power summation factor is 0.719 at $L / R=0.596$ and $L / R=0.384$. At the same time, there is a sharp drop in the power summation coefficient when the operating oscillation in the resonator interacts with the $\mathrm{TEM}_{309}$ oscillation $(L / R=0.49)$. At $L / R=0.225$, the drop-in $K_{\text {summ }}^{2}$ is due to the behavior of $K_{\text {transm }}^{(\mathrm{I})}, K_{\text {transm }}^{(\mathrm{II})}$ and $K_{\text {transm }}^{(\mathrm{III})}$. Thus, for the effective summation of the powers in the OR, only the operating mode of oscillations should be excited.

In order to understand what distances between the OR mirrors $(L / R<0.5$ or $L / R>0.5)$ are advised to use for the power summation of individual sources, it is necessary to determine the loaded $Q$-factors $Q_{L}$ for both indicated distances. This is due to the fact that the higher the $Q$-factor of the resonant system, the lower the phase noise level of the output sum signal [18]. Since we work at a fixed frequency, we will use the formula $Q_{L}=L / \Delta l$ [19] to find the loaded $Q$ factors $Q_{L}$ of the TEM ${ }_{1011} \quad(L / R=0.596)$ and $\mathrm{TEM}_{107}$ $(L / R=0.384)$ oscillations. Here $L$ is the resonant distance corresponding to the maximum value of transmission coefficient, $\Delta l=L_{1}-L_{2}$. The $L_{1}$ and $L_{2}$ values correspond to the distance between the resonator mirrors, at which the transmission coefficient decreases by $-3 \mathrm{~dB}$. As a result of the experimental studies, it has been found that the loaded $Q$ factor of the TEM 1011 oscillation is 528, and the loaded $Q$ factor of the TEM 107 oscillation is 332. Based on the above, we choose the distance between the resonator mirrors corresponding to the maximum value of the loaded $Q$ - factor of the operating oscillation. For the TEMo0q oscillation excited in a hemispherical OR, the mode of the maximum value of the loaded quality factor takes place at $L / R=$ $=0.7 \div 0.75[20,21]$, and for the $\mathrm{TEM}_{10 q}$ oscillation, this mode corresponds to $L / R \approx 0.6$ [22].

As shown above, the TEM 1011 oscillation mode corresponds to this distance between the mirrors of the resonator under consideration. Let us look at the structure of the field of this oscillation in the OR. To do it we use the test 
body method [17]. The field distribution will be measured using test body 20, fixed on nylon thread 21 (see Fig. 1 and Fig. 3). The diameter of the test body, which is a metal ball, is $1 \mathrm{~mm}$. The measurements are carried out in the plane of the $\mathrm{TE}_{10}$ wave vectors $\mathbf{H}$ in slotted coupling elements 18 , made on a flat mirror (see Fig. 4), in the first antinode of the standing wave electric field in the resonator, counting from the indicated mirror. The measurement results are shown in Fig. 6 (curve 1).

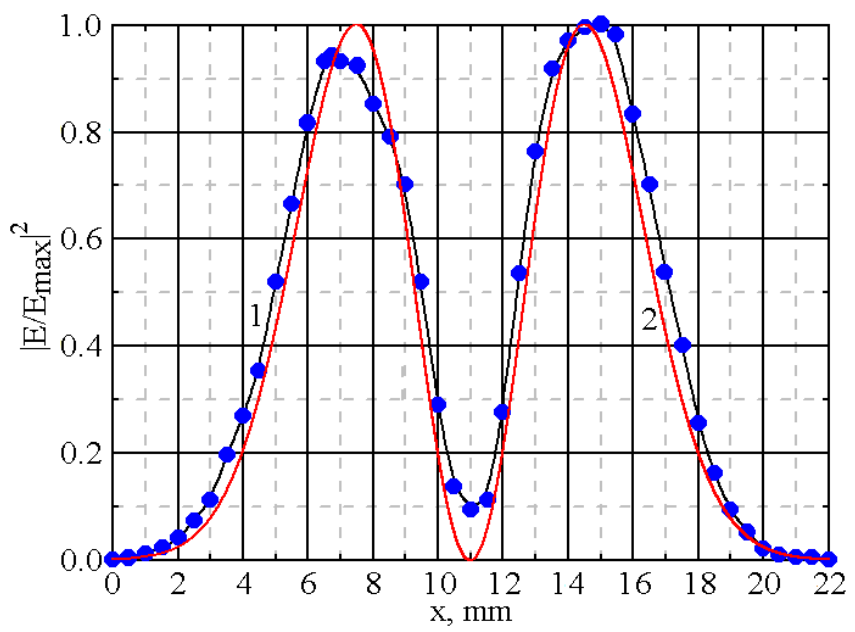

FIGURE 6. The structure of the TEM $\mathrm{T}_{1011}$ oscillation field in a hemispherical OR.

In order to construct the calculated distribution of the oscillation field $\mathrm{TEM}_{1011}$ on a flat resonator mirror, it is necessary to determine the resonance distance corresponding to the TEM0011 oscillation. This oscillation turned out to be excited in the considered OR at $L / R=0.58(R=39 \mathrm{~mm})$. To calculate the radius of the field spot $w_{0}$ of the TEM0011 oscillation on a flat mirror we use the formula [17]

$$
w_{0}=\sqrt{(\lambda / \pi) R \sqrt{(L / R)(1-(L / R))}} .
$$

Here $\lambda=4.003 \mathrm{~mm}$. After numerical value substitution into expression (1), we obtain that $w_{0}=4.953 \mathrm{~mm}$. To construct the calculated distribution of the TEM 1011 oscillation field on a flat resonator mirror, we use the expression

$$
\left|E / E_{\max }\right|^{2}=\left|\left(\sqrt{2 e} x / w_{0}\right) \exp \left(-\left(x / w_{0}\right)^{2}\right)\right|^{2} .
$$

The calculation results using formula (2) are shown in Fig. 6 (curve 2). From the given figure, we see a good agreement between the calculation and experiment. The relative error in the difference between the calculated value of the amplitude distribution of the field of the considered TEM $\mathrm{T}_{1011}$ oscillation and the measured one does not exceed $25 \%$. This difference is due to the following. As stated above, we use a $1 \mathrm{~mm}$ diameter scattering test body. The loaded $Q$ - factor of the oscillation under consideration is 528. It follows from [23] that for the indicated values of the loaded $Q$ - factor $Q_{L}$ and the resonant frequency $f$, the diameter of the test body should be $1.3 \mathrm{~mm}$. This is the reason for the difference between calculation and experiment.

\section{STRIP DIFFRACTION GRATING IN A HEMISPHERICAL OR}

As shown in [24], using a one-dimensional ribbon diffraction grating, placed in the OR volume perpendicular to its axis, it is possible to change the connection of the resonator to the supply waveguide path in a wide range of dimensions. In this case, it is advisable to use an $H$-polarized diffraction grating, since it causes significantly lower ohmic losses than an $E$ polarized grating [24]. The main mode of $\mathrm{TEM}_{00 q}$ oscillations of OR is under consideration in the paper. In our case, the first higher axially asymmetric mode of TEM $10 q$ oscillations is excited in the resonator. Therefore, analyzing the influence of a strip $H$ - polarized diffraction grating on the resonant transmission coefficient of a hemispherical OR, where the specified type of oscillation is excited, is of some practical interest. Consider $H$ - polarized diffraction grating 26 , the period of which is $s=0.8 \mathrm{~mm}$, the slit width is $d=0.12$ mm. A $37 \mathrm{~mm} \times 37 \mathrm{~mm}$ grating was made by photolithography from copper foil $\sim 20 \mu \mathrm{m}$ thick and fixed in mandrel 27 (see Fig. 7). The movement of the grating is controlled using micrometer head 28 with an accuracy of $0.01 \mathrm{~mm}$.

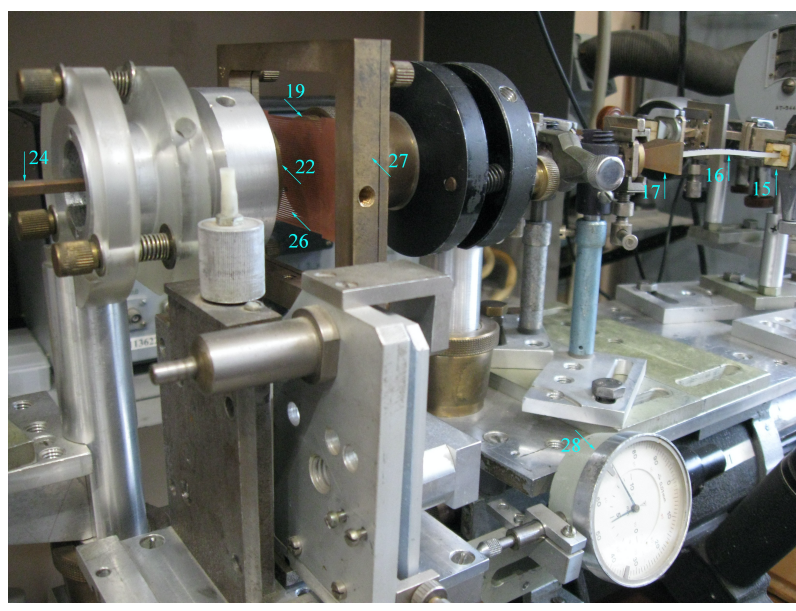

FIGURE 7. Ribbon $H$ - polarized diffraction grating in OR.

The grating is placed perpendicular to the OR axis in the first nodal plane $(a=0 \mathrm{~mm})$ counting from the plane mirror, where the electric field strength of the standing wave in the resonator is zero. The grating is phase transparency, when it is located near the specified mirror, the phase distortions introduced into the distribution of the operating mode of oscillations will be minimal. 
The resonator is excited using two slotted coupling elements made on a flat mirror (see Fig. 2). Let's consider the TEM $_{1011}$ oscillation in a hemispherical OR (see Fig. 6). The results of experimental studies are shown in Fig. 8, 9.

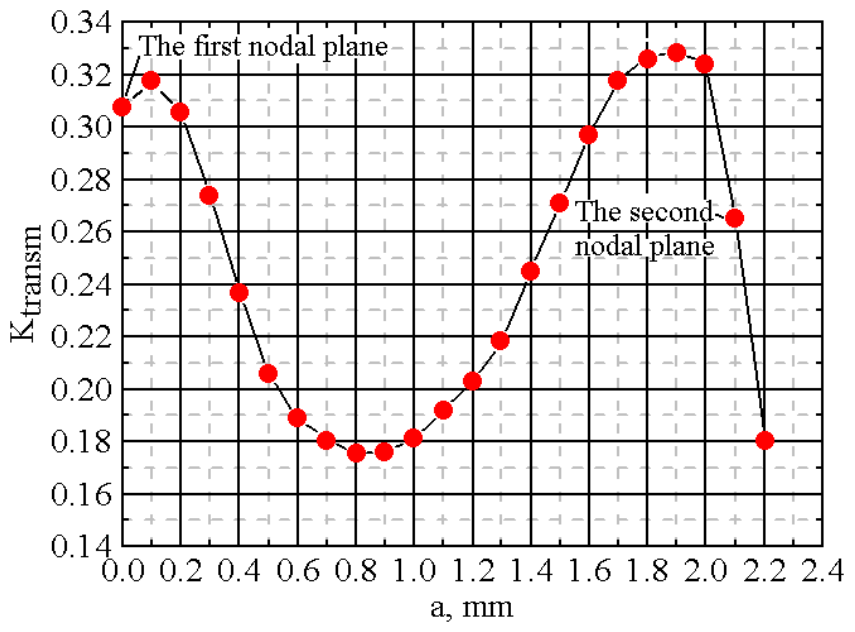

FIGURE 8. Dependence of the transmission coefficient of the OR on the grating location.

The measurement technique is as follows. As mentioned above, we place the grating in the cavity volume perpendicular to its axis in the nodal plane. In this case, we fix the resonant transmission coefficient. Now we shift the grating by $a=0.1 \mathrm{~mm}$ towards the spherical mirror and, by moving one of the resonator mirrors (in this case the flat one), we achieve the maximum value of the transmission coefficient. We fix the distance between the mirrors. Then we shift the grating in the same direction by $0.1 \mathrm{~mm}$ again and find the resonance distance corresponding to the maximum value of the transmission coefficient. This continues until the second nodal plane counting from the plane mirror, where the strength of the electric field of the standing wave in the resonator is zero.

As you can see from Fig. 8, when the grating is located in the first nodal plane, the transmission coefficient of the resonator is 0.308 . This value almost coincides with the transfer coefficient of the cavity $K_{\text {transm }}^{(\mathrm{I})}$ in the case of an empty cavity, equal to 0.302 (see Fig. 4 , curve $1, L / R=0.596$ ). Therefore, we can say that the grating in the resonator has focusing properties [24] within the various ranges of the parameter $a$ equal to $0 \mathrm{~mm} \div 0.2 \mathrm{~mm}$ and $1.65 \mathrm{~mm} \div 2.03$ $\mathrm{mm}$. This makes it possible to obtain a resonant transfer coefficient greater than the one in the case of an empty resonator. At $a=1.9 \mathrm{~mm} K_{\text {transm }}^{\max }=0.329$ (see Fig. 8). This value of the resonant transfer coefficient is only $9 \%$ higher than $K_{\text {transm }}^{(\mathrm{I})}$ for an empty cavity $(0.302)$. Thus, we can say that in the case of a slotted excitation method, a onedimensional ribbon $H$-polarized diffraction grating does not allow a significant increase in the resonance transmission coefficient and, as a consequence, the power summation coefficient in a hemispherical OR. The aforesaid refers to the excitation of the first higher axially asymmetric type of $\mathrm{TEM}_{1011}$ oscillations in the resonator.

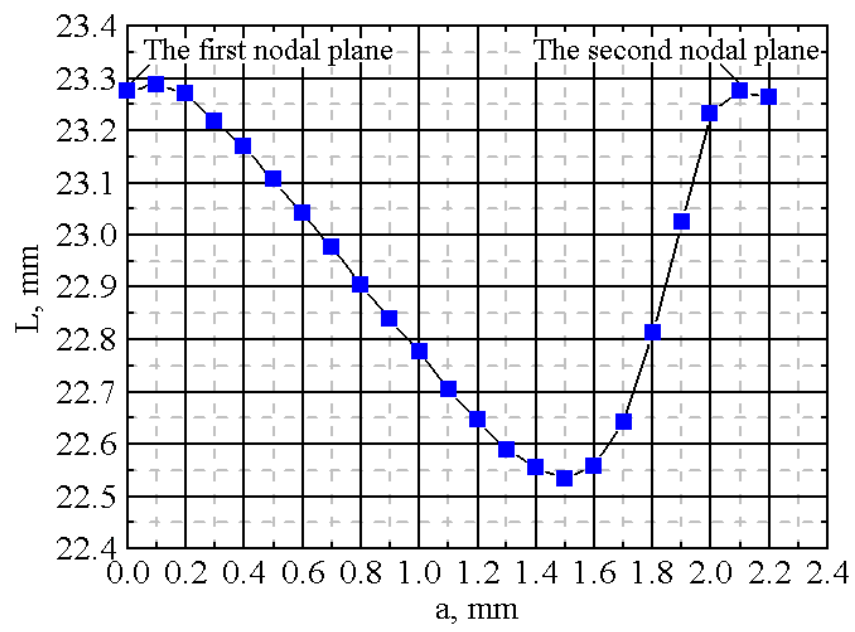

FIGURE 9. Dependence of the distance between the OR mirrors on the grating location.

The ribbon grating in the resonator leads to defocusing the Gaussian beam in the various ranges of the parameter $a$ equal to $(0.2 \mathrm{~mm} \div 1.65 \mathrm{~mm})$ and $(2.03 \mathrm{~mm} \div 2.2 \mathrm{~mm})$. In this case, the resonant transfer coefficient decreases in comparison with an empty OR [24].

Fig. 9 shows the dependence of the distance $L$ between the resonator mirrors on the position of the tape $H$-polarized diffraction grating in its volume for the TEM 1011 oscillation. It can be seen from the figure that when the grating possesses focusing properties, the resonance distance between the mirrors increases $(a=0 \mathrm{~mm} \div 0.2 \mathrm{~mm})$ compared to an empty OR. When the grating has defocusing properties, the distance between the resonator mirrors decreases $(a=0.2 \mathrm{~mm} \div 1.5 \mathrm{~mm})$.

\section{CONCLUSION}

The experimental studies carried out in this work enable us to draw several important practical conclusions.

1. Using an $E$ - tee waveguide, it is possible to study the power summation of two sources in an OR in which the first higher axially asymmetric mode of $\mathrm{TEM}_{10 q}$ oscillations is excited. In this case, only one Gunn diode is used.

2. The maximum coefficient of power summation of two sources in OR with two slot coupling elements is 0.719 . This result is in good agreement with the data of [14]. Thus, it is impossible to obtain the power summation coefficient of the two sources higher than 0.8 using a slot method of resonator excitation.

3. To obtain the maximum power summation coefficient in the resonator only the operating mode of oscillations should be excited. The distance between the resonator mirrors should correspond to the maximum value of the loaded $Q$ factor of the operating oscillation to reduce the phase noise of the output sum signal. 
4. In the case of a slotted excitation method, the location of a strip $H$ - polarized diffraction grating in the cavity volume does not lead to a significant increase in the transmission coefficient of the oscillation considered and, as a consequence, in the power summation coefficient.

\section{REFERENCES}

[1] P.H. Siegel, "Terahertz Technology," IEEE Trans. on Microwave Theory and Techniques, vol. 50, no, 3, pp. 910-928, March 2002, DOI: $10.1109 / 22.989974$

[2] G. Sitnikov, V.M. Mikhailov, and A.E. Telminov, "Terahertz Radiation: Applications in Molecular Spectroscopy and Sources of Molecular Radiation," Atmospheric and Oceanic Optics, vol. 22, no. 11, pp. 1092-1098, Nov. 2009. (In Russ.).

[3] Z. Popovic, and E.N. Grossman, "THz Metrology and Instrumentation," IEEE Trans. on Terahertz Science and Technology, vol. 1, no. 1, pp. 133-144, Jan. 2011, DOI: 10.1109/TTHZ.2011. 2159553.

[4] G.N. Kulipanov, "Generation and Application of Terahertz Radiation: History and Perspective," Vestnik Novosibirsk State University. Series: Physic, vol. 5, no. 4, pp. 24-27, April 2010. (In Russ.).

[5] A. Svetlitza, M. Slavenko, T. Blank, and et al., "THz Measurements and Calibration Based on a Blackbody Source," IEEE Trans. on Terahertz Science and Technology, vol. 4, no. 3, pp. 347-359, March 2014, DOI: 10.1109/ TTHZ. 2014.2309003.

[6] N.F. Karushkin, L.V. Kasatkin, and S.B. Malcev, "Experience in Development of High Power IMPATT Diode Sources for MM-Wave Range," 16th International Crimean Conference "Microwave \& Telecommunication Technology" (CriMiCo'2006), vol. 1, pp. 135137, Sept. 2006, DOI: 10.1109/ CRMICO. 2006.256331.

[7] S. Yatsenko, O. Litvin, O. Litvin, and N. Shumejko, "Application of Terahertz Radiation for Nondestructive Testing of Quality of Products," International Conference "NDT Days 2016", pp. 67-70, June 2016, DOI: search/docs.php3?id=20477.

[8] A.I. Borodkin, B.M. Bulgakov, V.A. Matveeva, and et al., "Millimeter-range semiconductor source with quasi-optical resonator," Technical Physics Letters, vol. 5, no. 5, pp. 285-288, March 1979. (In Russ.).

[9] S.M. Ovechkin, S.I. Rebrov, V.P. Sazonov, and et al., "Power Summation of the Gunn Diodes in an Open Microwave Resonator," Technical Physics Letters, vol. 10, no. 6, pp. 367-370, March 1984. (In Russ.).

[10] K. Mizuno, T. Ajikata, M. Hieda, and M. Nakayama, "Quasi-Optical Resonator for Millimetre and Submillimetre Wave Solid-State Sources," Electronics Letters, vol. 24, no. 13, pp. 792-793, June 1988, DOI: 10.1049/el:19880538.

[11] J. Bae, Y. Aburakawa, H. Kondo, and et al., "Millimeter and Submillimeter Wave Quasi-Optical Oscillator with Gunn Diodes,“ IEEE Trans. on Microwave Theory and Techniques, vol. 41, no. 10, pp. 1851-1855, Oct. 1993, DOI: 10.1109/22.247932.

[12] R. Judaschke, M. Hoft, and K. Schunemann, "Quasi-Optical 150GHz Power Combining Oscillator," IEEE Microwave and Wireless Components Letters, vol. 15, no. 5, pp. 300-302, May 2005, DOI: 10.1109/LMWC.2005.847660.

[13] A.A. Dvornikov, and G.M. Utkin, "On the Addition of the Powers of Many Auto Generators," Radiotehnika i Elektronika, vol. 19, no. 3, pp. 550-559, March 1974. (In Russ.).

[14] R.K. Tyagi, and D. Singh, "Quasi-Optical Resonator for Power Combining at W-Band," Int. J. of Infrared and Millimeter Waves, vol. 17, no. 2, pp. 385-391, Feb. 1996, DOI: 10.1007/BF02088161.

[15] A.V. Arkhipov, O.I. Belous, B.M. Bulgakov, and A.I. Fisun, "Millimeter Wave Power Combiner Based on a Half-Open Resonator," Int. J. of Infrared and Millimeter Waves, vol. 23, no. 3, pp. 507-516, March 2002, DOI: 10.1023/A:1015054124268.

[16] A.S. Shlapakovski, S.N. Artemenko, P.Yu. Chumerin, Yu.G. Yushkov, "Formation of Pulses with Adjustable Parameters in a Resonant Microwave Pulse Compressor," Advanced Materials Research, vol. 1084, pp. 256-261, Sept. 2015, DOI:10.4028/www.scientific.net/AMR.1084.256.
[17] R. A. Valitov, and V. V. Kamyshan, "Resonators," in Submillimeter Wave Technique, R. A. Valitov, Ed. Moscow: Soviet Radio Press, 1969, pp. 219-229. (In Russ.).

[18] A. Chenakin, "Microwaves Generators Phase Noise. Methods for Problems Solution," Elektronika: Nauka. Tekhnologiya. Biznes, no. 4, pp. 52-61, June 2011. (In Russ.).

[19] Y. Zhonghai, L. Chongwen, and Z. Yingwei, "A Method for Measurement of Q-Factor at Millimeter Wavelength," 10th International Conference on Infrared and Millimeter Waves, pp. 350351, Dec. 1985, DOI: 10.1109/IRMM.1985.9126718.

[20] A.V. Arkhipov, I.K. Kuzmichev, I.I. Reznik, and D.G. Seleznyov, "Microstrip Grid in Open Resonator," Physics of Wave Processes and Radio Systems, vol. 7, no. 1, pp. 7-11, March 2004. (In Russ.).

[21] R.F. Soohoo, "Nonconfocal Multimode Resonators for Masers," Proc. of the IEEE, vol. 51, no. 1, pp. 70-75, Jan. 1963, DOI: 10.1109/PROC.1963.1661.

[22] A.V. Arkhipov, O.I. Belous, I.K. Kuzmichev, and A.S. Tishchenko, "Quasi-Optical Resonance System for Solid-State Oscillator," Telecommunications and Radio Engineering, vol. 61, no. 6, pp. 476484, June 2004, DOI: 10.1615/TelecomRadEng.v61.i6.30.

[23] I.K. Kuzmichev, "The Probe Diameter Choosing for the Investigation of the Field Distribution in the Small Aperture Open resonator," Telecommunications and Radio Engineering, vol. 58, no. 1, pp. 59-63, Jan. 2002, DOI: 10.1615/TelecomRadEng.v58.i7-8.50.

[24] V.P. Androsov, and I.K. Kuzmichev, "Influence of internal inhomogeneities of an open resonator on the coupling of its oscillations with waveguide lines," Kharkov, Institute for Radiophysics and Electronics of AS USSR, Preprint, no. 355, 14 p., 1987. (In Russ.). 